Linguistique, littérature, didactique

157-158 | 2013

Théories et pratiques des genres

\title{
Sémiotique textuelle du genre
}

La généricité des albums d'enfance

\section{Nicolas Couégnas}

\section{(2) OpenEdition}

Journals

\section{Édition électronique}

URL : http://journals.openedition.org/pratiques/3671

DOI : 10.4000/pratiques.3671

ISSN : 2425-2042

Éditeur

Centre de recherche sur les médiations (CREM)

Édition imprimée

Date de publication : 1 juin 2013

Référence électronique

Nicolas Couégnas, « Sémiotique textuelle du genre », Pratiques [En ligne], 157-158 | 2013, mis en ligne le 18 décembre 2017, consulté le 01 mai 2019. URL : http://journals.openedition.org/pratiques/3671 ; DOI : 10.4000/pratiques.3671 


\section{Sémiotique textuelle du genre. La généricité des albums d'enfance}

\section{Nicolas Couégnas}

CeReS. Université de Limoges

Les albums d'enfance présentent la caractéristique, et l'intérêt, de rendre à peu près incontournable la prise en compte du point de vue interprétatif. Ignorer, pour ce corpus, les spécificités de la production et de l'interprétation reviendrait à tourner délibérément le dos à tout principe d'empirisme. Les albums d'enfance s'adressent à un public particulier, qui reçoit ces albums dans des conditions également particulières, et dans des formes tout aussi spécifiques. Concrètement : les albums visent un public d'enfants non lecteurs, ils sont « lus », la plupart du temps, dans une situation de lecture inédite, qui voit un adulte ou au moins un lecteur, lire le texte et commenter les images de l'album à un enfant qui écoute et observe les images. Autrement dit, les albums, comme tout texte présentant une visée pragmatique précise, ne souffrent aucune réduction immanentiste, si immanence signifie, dans une acception restreinte, suspension du contexte ou de la pratique interprétative associée au texte.

C'est assez naturellement, dans ce cadre, que la question du genre émerge. On peut en effet s'attendre à ce qu'à la spécificité du format de réception fasse écho une relative stéréotypie des formes utilisées. Et en effet, malgré la qualité générale des ouvrages, la diversité de l'offre et la bonne santé du secteur éditorial, l'air de famille, d'un ouvrage à l'autre, devient presque obsédant dès lors qu' on y prend garde. Dès qu'on s'est penché sur le socle générique des albums d'enfance, on ne cesse de le voir à l'œuvre et d'en observer les déclinaisons possibles dans la diversité de la production. De l'air de famille à la question du genre il n'y a qu'un pas, que l'on peut franchir sans peur ou refuser aussi bien selon l'idée et la définition que l'on se donne du genre. On s'accordera a minima sur le constat d'une forte généricité qui semble peser sur les albums d'enfance. Ce ne sont pas tous les mêmes, mais ils ont tous quelque chose en commun!

Ce constat engage plusieurs questions, auxquelles nous répondons en introduisant trois concepts, qui établissent les fondements d'une sémiotique textuelle $d u$ genre: 1) versant théorique, le fait de poser la reconnaissance, empirique, d'un air 
de famille conduit à adopter une conception du genre bien définie, où le genre est conçu comme forme de généricité ensynchronie ; cette position n'exclut pas d'autres possibilités, valables sans doute dans d'autres perspectives d'analyses, mais elle est dictée par le principe d'empirisme suivi ; 2) ce point de vue synchronique a des conséquences : il implique que le genre doit avoir une traduction pleinement textuelle, systématique, avec une incidence à la fois sur la génération et l'interprétation du texte. La définition sémantique du genre, donnée par F. Rastier ${ }^{(1)}$ permet précisément d'appréhender le genre comme une composante textuelle à part entière, dont le pouvoir de coercition sur les œuvres n'est pas hypothétique ou extérieur aux productions textuelles mais directement manifestés par les textes analysés. Cette approche sémantique du genre aboutit, pour les albums d'enfance, à ce que l'on définira comme un arbre générique, où s'exprime la hiérarchisation entre composantes textuelles imposée par le genre (3) enfin la généricité ouvre des perspectives sur son envers : la singularité des textes, qu'il convient de penser à partir des contraintes du genre inscrites dans un gradient de généricité. De même que pour le concept d'arbre générique, la gradualité des contraintes génériques conduit à proposer des articulations à partir des définitions de Rastier, et en partie à redéfinir ces premières définitions.

\section{Le genre comme généricité synchronique}

Tous les lecteurs d'albums, parents, enfants ou professionnels du livre, ont une idée relativement précise de ce qu'est un album, ils savent aussi ce que n'est pas un album, et les difficultés que l'on rencontre lorsque l'on s'essaye à lire une bande dessinée à un enfant. Et pourtant, si l'on peut dater l'apparition de l'album dans sa forme actuelle et le définir, en l'opposant notamment à ses plus proches cousins, tels la bande dessinée, les imagiers et autres textes de la littérature d'enfance et de jeunesse associant texte et image, la reconnaissance d'un genre à part entière semble faire problème. Sophie Van der Linden ${ }^{(2)}$ note que le genre de l'album est problématique parce qu'il emprunte des normes textuelles à plusieurs traditions : contes, policiers, poésie, autant de sources d'inspiration et de structures reconnaissables susceptibles de le traverser pour lui donner sa forme particulière. Pour rendre compte néanmoins de l'air de famille évident des albums, de leur homogénéité et de leur réussite fonctionnelle, nous utilisons le concept de gradient de généricité.

En premier lieu, la notion de généricité permet de se démarquer du genre au sens un peu encombrant où l'entend Sophie Van Der Linden, qui lui interdit de prendre en compte la ressemblance globale de ces œuvres particulières ainsi que les critères génériques précis qui font qu'un album est reconnaissable et exploitable en tant qu'album pour enfant. Il y a, peut-être, dans cette démarche la volonté de refuser de circonscrire trop drastiquement les albums et la peur que cette réduction fasse office de déni de créativité ou de refus de littérarité. Ce à quoi on peut objecter que la généricité ne contredit pas la singularité si l'on admet que la généricité est graduelle et qu'elle s'exprime comme un ensemble de contraintes qui ne laisse en rien présumer du contenu et de la forme finale. Seconde objection, plus immé-

(1) Par exemple dans Arts et sciences des textes, Paris, PUF, 2001, ou encore dans «Éléments de théorie des genres », Texto ! juin 2001 [en ligne]. Disponible sur : ttp ://www.revue-texto.net/Inedits/Rastier/Rastier_Elements.html. (Consultée le 25-05-2012).

(2) Van der Linden S. 2006. 
diatement pertinente, il semble que l'on confonde, avec l'argument de l'hétérogénéité générique, avancée notamment par la spécialiste des albums d'enfance, entre genres inclus et genre englobant. L'inspiration policière, poétique, etc. nourrit sans conteste les textes, et fixe également des contraintes textuelles, mais qui n'interviennent pas au même niveau : elles prennent effet au sein des formes préalablement dictées par les lois du genre. L'album reste un album, même s'il prend des allures d'album policier ou d'album poème.

En second lieu, l'expression « air de famille» a été employée dans un sens non technique, mais il y a beaucoup plus qu'un simple cousinage entre les albums. Dit autrement, là où l'air de famille ne décrirait qu'une stabilité de formes, plus ou moins facilement identifiable d'un album à l'autre selon le principe de l'air de famille, la généricité, dans le sens où nous l'utilisons, signifie une dynamique, une force textualisante. Ce terme de généricité est utilisé par Jean-Michel Adam et Ute Heidmann, dans un article intitulé : Des genres à la généricité : L'exemple des contes (Perrault et les Grimm) ${ }^{(3)}$ dans un sens différent, mais où la dimension dynamique est également bien présente :

«L'étiquette genre et les noms de genres — «conte de fées », « Märchen », « tragédie », « fait divers », etc. — ont tendance à réduire un énoncé à une catégorie de textes. La généricité est, en revanche, la mise en relation d'un texte avec des catégories génériques ouvertes $»{ }^{(4)}$

ou encore :

«Le passage du genre à la généricité est un changement de paradigme. La mise en relation d'un texte considéré dans sa clôture avec une catégorie générique constituée généralement en essence diffère profondément de la dynamique socio-cognitive que nous nous proposons de mettre en évidence $» .{ }^{(5)}$

On retrouve ici l'argument du bénéfice du concept de généricité par rapport à l'étiquette, statique, du genre. La généricité s'oppose ici à la clôture textuelle et à la définition littéraire classique d'un genre défini « en essence » et introduit, littéralement, une dimension dynamique, qualifiée par les auteurs de « socio-cognitive ». Nous conservons la composante dynamique que peut porter le terme de généricité mais dans une autre direction, plus conforme aux propositions sémantiques de Rastier. En lieu et place d'une force socio-cognitive, qui fait signe vers deux extérieurs du texte, nous posons l'existence d'une force générique interne, dont le texte est la manifestation. Pour le dire de manière plus précise, et sans position inutilement polémique, la généricité est une force, une dynamique dont on peut soit rechercher les mobiles extérieurs, soit les effets textuels.

Reste qu'une différence forte demeure entre les deux points de vue, qui se résume essentiellement à l'opposition fondamentale entre diachronie et synchronie. Dans leur article, Adam et Heidmann, s'attaquent en effet, au moins dans la première partie, de manière très intéressante, à une description essentiellement diachronique du genre du conte. Dans cet exercice tout à fait passionnant, et tout à la passion de cet exercice, ils écrivent de manière très polémique, au moins du point de vue d'une sémiotique et d'une sémantique d'inspiration saussurienne :

(3) Jean-Michel Adam, Ute Heidmann, 2004.

(4) Op. cit., p. 6.

(5) Op. cit., p. 62-63. 
« comme pour la langue, la codification en synchronie stable d'un genre est un effet trompeur de la coupe analytique pratiquée dans un continuum dynamique ${ }^{(6)}$. Sans entrer ici dans la discussion, remarquons simplement que la seconde partie de leur article, sur la textualité, mobilise une analyse synchronique ; cette partie reste générale et programmatique, mais en affirmant que le genre a une incidence sur tous les niveaux de la textualité, elle exprime parfaitement la nécessité d'une analyse synchronique. La dynamique générique que nous postulons adopte ce point de vue synchronique, pour montrer, dans ce cas précis, comment la généricité de type album d'enfance agit réellement sur les composantes textuelles.

De plus, pour Adam et Heidmann, en raison de la perspective diachronique adoptée, il y a une hétérogénéité générique des textes, qui sont le lieu de tensions entre des généricités auctoriale, lectoriale et éditoriale. Nous nous rangeons à la critique de Rastier, qui considère au contraire qu'un texte appartient nécessairement d'abord à un genre particulier, condition de sa lisibilité et de son interprétation. Mais la question a déjà été évoquée de la coexistence de ces deux modes d'existence du texte et de leur complémentarité : les ajustements et les transformations génériques de la diachronie n'excluent nullement la pertinence de la visée synchronique, et inversement.

\section{L’arbre générique}

\section{Le genre comme interaction entre les composantes textuelles}

Si le genre est bien une catégorie efficiente en synchronie, un pouvoir de structuration dont le texte est la manifestation, on doit donc pouvoir le définir à partir des seules composantes de la textualité. L'approche de François Rastier satisfait à cette exigence synchronique. Conformément aux principes de sa sémantique étendue, qui reconnaît, outre la langue, que plusieurs instances de systématicité sont à l'œuvre dans un texte, il affirme tout d'abord qu' " un genre est un programme de prescriptions positives ou négatives, et de licences qui règlent aussi bien la génération d'un texte que son interprétation », ensuite que ces prescriptions « ne relèvent pas du système fonctionnel de la langue, mais d'autres normes sociales ${ }^{(7)}$. Le genre apparaît donc comme un ensemble de contraintes qui s'exercent dans le texte, comme instance de systématicité, au même titre que la langue. Il faut que la sémantique soit capable de faire place à cette dimension qui excède la langue pour devenir capable de saisir les influences génériques en synchronie. Rastier ajoute que « aux trois paliers que nous avons distingués [nous rajoutons : mot, phrase, texte], et plus particulièrement au dernier, un genre se définit par l'interaction de normes socialisées, dans plusieurs des composantes linguistiques (du contenu comme de l'expression) $\gg{ }^{(8)}$. Le genre est susceptible de peser sur chacun des paliers du texte : par exemple, le lexique et la syntaxe des albums d'enfance sont évidemment très contraints par le genre « album d'enfance », et n'y pas obéir rend les albums à peu près inaudible pour les enfants. Mais le lieu d'expression privilégié du genre reste le niveau textuel, dans sa globalité, qui seul permettra en retour de le définir de manière synthétique. Concrètement, le genre se définit alors, en sui-

(6) Op. cit., p. 62-63.

(7) Rastier $1994: 222$

(8) Rastier 1989:106-107. 
vant Rastier, par un type d'interaction entre composantes au sein des plans du contenu et de l'expression, ainsi qu'entre ces deux plans. Rastier répartit les différentes composantes du plan de l'expression et du plan du contenu comme suit :

\begin{tabular}{|l|l|}
\hline Composantes du signifié & Composantes du signifiant \\
\hline Thématique (thèmes, isotopies, topos) & Médiatique (écrit, oral, polysémiotique) \\
Dialectique (narrativité) & Rythmique \\
Dialogique (énonciation) & Prosodique-tonale \\
Tactique (disposition/organisation du plan & Distributionnelle \\
du contenu) & \\
\hline
\end{tabular}

En faisant un peu violence aux catégories de Rastier, on retiendra que la thématique correspond aux contenus (thème, isotopies, topos), la dialectique à la narrativité, la dialogique à l'énonciation et la tactique à l'organisation et à la disposition des unités du plan du contenu. Pour le plan de l'expression, les albums investiront tout particulièrement la composante médiatique, dont relève tout ce qui concerne les supports et matière du plan de l'expression, et la composante distributionnelle, pendant de la tactique pour le plan de l'expression; elle correspond à l'organisation des unités du plan de l'expression dans la linéarité textuelle et au sein de la page du texte. Ces propositions de Rastier donnent une traduction très concrète au pouvoir de coercition du genre et à sa manifestation textuelle synchronique : un genre particulier spécifiera telle ou telle composante et privilégiera la mise en relation de telle ou telle composante.. Nous ajoutons, au moins pour le cas de l'album, l'idée que le genre produit une hiérarchisation des composantes textuelles, le genre s'appréhendant alors comme un arbre générique.

\section{Le socle générique de l'album d'enfance ${ }^{(9)}$}

L'album est d'abord spécifique en raison de sa composante médiatique, qui le rend immédiatement reconnaissable. Un album tisse textes et images en accordant, la plupart du temps, un rôle prépondérant à l'image. Le très beau Macao et Cosmage marque d'ailleurs en 1919 la naissance de l'album ${ }^{(10)}$ parce qu'il accomplit une révolution médiatique, jusqu'alors rendue impossible en raison des limites imposées par les techniques d'impressions (2006, Van der Linden) : il exploite l'iconicité de l'image, qui sort ainsi d'un long cantonnement dans un rôle illustratif. Une composante médiatique nouvelle, propre à l'album, est créée, qui se laisse principalement résumer par le rapport texte/image, avec une forte présence de l'image impliquant très souvent une supériorité sur le texte, qui peut d'ailleurs aller jusqu'à l'effacement de ce dernier Un album sans texte reste un album à part entière, peut-être même touche-t-il à l'essence de l'album, alors qu'un album sans image devra se contenter du statut de simple histoire.

Cette évolution semble incontournable et irréversible, notamment parce

(9) Nous reprenons une analyse déjà présentée synthétiquement dans «L'art de la surprise et de la répétition dans la littérature d'enfance et de jeunesse », dans Répétition et habitude dans les pratiques quotidiennes, dirigé par J. Alonso et E. Bertin, revue Protée, Université du Québec à Chicoutimi, volume 38, n², Automne 2010.

(10) Michel Defourny, préface à l'édition de 2000 de Macao et Cosmage (cité par Sophie Van der Linden, 2006, p. 11) : «Voici en effet une œuvre qui privilégie explicitement le visuel, annonçant, dès 1919, l'album contemporain pour enfants ». 
qu'elle colle parfaitement à la situation de lecture et d'interprétation. L'enfant ne sachant pas lire, c'est logiquement l'iconicité de l'image qui se trouve sollicitée, son pouvoir de dire le monde sans béquille verbale. L'image ouvre un accès en partie direct pour l'enfant au sens de l'histoire. Cette évolution traduit d'ailleurs un changement social profond puisqu'il implique l'idéal d'une autonomisation de l'enfant qui n'est plus uniquement réduit à deux oreilles mais se voit également doté d'une paire d'yeux et d'un esprit qui participe à la reconstruction de l'histoire. Le rôle accordé à l'image crée une interaction et un partage entre le lecteur adulte et l'enfant, et donc une relative co-construction du sens. Ce qui est d'autant plus vrai que l'image, si elle parle, n'est pas univoque et offre une plus ou moins large palette de points de fixation et d'interprétations. On peut aussi imaginer que l'accès iconique au sens produit une meilleure fixation de l'attention et une meilleure compréhension.

Plus surprenant, ce socle médiatique incontournable, parfaitement adapté à la situation de lecture, va également avoir une incidence très forte sur toutes les autres composantes sémantiques : la distribution (expression) et la disposition (composante tactique, du contenu) mais aussi la narration et le point de vue. Autrement dit c'est le rapport texte/image de l'album qui va déterminer en grande partie les ressorts textuels typiques des albums. C'est là que réside le noyau générique de l'album : dans la force de la composante médiatique dont les effets se font sentir de manière spectaculaire dans les différentes composantes sémantiques du texte.

Le poids de 1'image est tout d'abord associé à une distribution du plan de 1'expression des albums : il y a toujours réduction des images à une image ou deux par page, la moitié d'une image lorsqu'il s'agit d'une page double, technique très souvent exploitée par les albums, deux images dans quelques cas peu fréquents, et très rarement plus. Le nombre réduit d'image par page est une nouvelle fois en harmonie avec le rôle dévolu à l'iconicité et permet à celui-ci de jouer à plein pour l'enfant qui observe, ce qu'interdirait la multiplication des images par page. L'image qui s'étale en pleine ou double page reste présente relativement longtemps, laissant à l'enfant le temps de la parcourir ; fonctionnant comme un bloc autonome, elle requiert moins de mémoire qu'une suite de vignettes. Elle réclame enfin moins d'inférences narratives puisqu'elle se donne à lire comme une petite totalité. L'album s'oppose en cela de manière très frappante à la profusion possible des vignettes dans une bande dessinée. La scansion des vignettes apparente plus la bande dessinée à un art du temps qu'à un art de l'espace, et c'est sans doute ce qui en rend la lecture si laborieuse à de jeunes enfants.

Jusqu'à présent, rien d'absolument surprenant. Ce qui l'est beaucoup plus, c'est la façon dont la disposition du plan de l'expression semble déterminer tout d'abord la disposition (tactique) du plan du contenu, puis les composantes du récit et du point de vue. En règle générale la linéarité du texte, qui fait se succéder les mots, ne présume en rien de la disposition du contenu : une unité sémantique peut apparaître au début ou à la fin du texte sans que la linéarité y soit pour grand chose. Ainsi le roman policier privilégie-t-il souvent l'exposé du crime en début de roman, la linéarité se mettant alors au service d'une reconstitution ou d'une rétrospection. La linéarité offre donc simplement des places à occuper dans la chaine textuelle. Or dans le cas de l'album, la disposition du contenu dépend très précisément de la disposition du plan de l'expression. Il faut expliquer ce point central et extrêmement productif dans le genre de l'album avec un exemple : La piscine (Poussier, 2008). Dans cet album (cf. annexe), une souris invite un lapin rose à plonger dans la piscine, mais ce- 
lui-ci refuse en prétextant la peur. La souris, sportive, se dirige vers le plongeoir dans la partie gauche de la double page afin de montrer au lapin rose que ce n'est pas si difficile. Sur la double page suivante le lapin n'a pas toujours pas bougé du côté gauche de la piscine, d'où il fixe avec attention le plongeoir dans la partie droite. La souris est dans l'air, elle vient de sauter et derrière elle se présente un gros chat gris en maillot de bain rétro, en train d'escalader l'échelle du plongeoir. Vient ensuite le tour d'une énorme poule rouge, puis du renard, puis de l'ours, puis du mouton, jusqu'à l'éléphant qui rejoint par la voie des airs l'ensemble des autres animaux, en train de barboter dans l'eau. La piscine est pleine, le lapin se plaint alors qu'il ne peut plus plonger et sauve ainsi sa dignité de lapin. Dans cet exemple classique, la progression de l'histoire implique nécessairement une lecture linéaire ; sans cette linéarité qui fait se succéder les doubles pages l'histoire perd tout son sens et toute sa saveur. L'augmentation de la taille des personnages, l'augmentation du nombre d'acteurs dans la piscine et par conséquent, au final, la diminution de la place dans la piscine seraient illisibles dans un autre ordre. Chaque contenu a besoin, pour être interprétable, d'apparaître page après page, au fil d'une linéarité sans faille. Sous cette progression linéaire du contenu, typique des albums, sont à l'œuvre une nouvelle fois la situation de lecture et d'interprétation et la composante médiatique : le lecteur regarde une page, un moment, avec l'enfant ; puis le changement de page intervient, produisant un changement de contenu, dont on suppose qu'il doit être suffisamment lié à la page précédente pour être interprété correctement par l'enfant. À la linéarité caractéristique de l'expression correspond terme à terme une linéarité du contenu tout aussi marquée, forme de l'expression et forme du contenu étant ici absolument indissociables.

Cette symétrie, qui peut apparaître de façon plus masquée dans certaines œuvres, constitue l'une des clés de la structure des albums exploitée par les trois grands modèles d'albums. Chaque modèle d'album va d'ailleurs apparaître comme une manière de répondre et de tenir compte au mieux de la linéarité de l'expression et du contenu, en transformant la contrainte générique en un puissant ressort textuel. On voit donc se dessiner une hiérarchie des composantes constitutives du genre de l'album. Le socle repose sur la composante médiatique. (texte/image, supériorité de l'image). Cette composante agit d'abord sur la disposition du plan de l'expression (faible nombre d'image par page ou double page). Cette disposition se répercute ensuite sur le plan du contenu, qui colle à la linéarité de l'expression.

\section{Socle générique des albums d'enfance :}

Médiatique $>$ Distribution de l'expression $>$ Tactique du contenu

Il faudrait également prendre en compte la composante thématique, typique des albums. Christian Chelebourg et Francis Marcoin (2007) observent que le thème central des albums est tout simplement l'enfant. L'écriture de l'enfance se traduisant par la reconstruction romanesque de l'univers de l'enfant, la transposition héroïque, les blessures de l'affect propres aux enfants, l'anthropomorphisme ou l'adoption du style « roman de formation » ${ }^{(11)}$. À partir de ce tronc commun on peut

(11) Nous ne développons pas ce point, central dans nombres d'étude (Chelebourg, Marcoin 2007), pour ne pas alourdir le propos. Ajoutons que le thème peut interagir avec toutes les autres composantes mais qu'il est plus difficile d'établir une hiérarchie. 
considérer que l'arbre générique se ramifie en plusieurs branches correspondant aux composantes restantes : le récit et le point de vue, qui vont donner leur forme aux trois grands modèles d'albums.

\section{Trois modèles pour les albums}

Entre le socle générique et les œuvres particulières nous distinguons des modèles intermédiaires, qui s'offrent aux créateurs comme une grammaire de formes malléables. Les deux premiers modèles, qui informent un très grand nombre d'album, relèvent, comme La piscine, de la progression narrative. Mais l'on doit distinguer entre un modèle narratif classique, où la structure narrative prime sur la mise en forme verbale et iconique, et un modèle qui fonde véritablement sa dynamique narrative sur le pouvoir des images. Le premier modèle narratif est onomasiologique : il va du contenu narratif à sa mise en signe, alors que le second peut être considéré comme sémasiologique parce qu'il va des signes et à leur pouvoir expressif au contenu. Le premier modèle sera dénommé simplement «Modèle du conte» et le second: «Modèle de la boule de neige ». Le troisième type d'album, également omniprésent, exploite les ressources du point de vue. Il est un maître de la rupture de perspective et des surprises, qui jouent sur des renversements, sur des retournements, des révélations, des tensions entre monde factuel et contrefactuel. Ce troisième modèle est baptisé " modèle de la surprise ». Il faut insister sur le fait que les albums concrets puisent dans les différents modèles, et plus rarement dans un seul. On peut donc classer les albums, quand la hiérarchisation est possible, dans telle ou telle famille en fonction du modèle dominant, sachant que les autres ne sont jamais très loin.

\section{1) Modèle du Conte (dialectique onomasiologique)}

Dans le modèle du conte, si l'image reste quantitativement prépondérante, l'histoire est néanmoins pensée à partir de la progression textuelle verbale. En d'autres termes, la progression de l'histoire ne doit rien à ce que l'image pourrait dire spécifiquement. En général l'image joue un rôle illustratif, soit en choisissant de représenter telle ou telle phase racontée par l'histoire, soit en représentant une phase qui n'est pas explicitement verbalisée mais que l'on peut reconstruire par inférence à partir du texte. La série des $T$ ' choupi exemplifie parfaitement ce modèle du conte dans sa version morale et édifiante. Le cœur de ce type d'album réside dans la narration, au demeurant très classique. L'analyse de plusieurs T'choupi montrerait qu'on retrouve toujours les mêmes classes d'acteur jouant les mêmes rôles : T'choupi, la tentation, les risques, les protecteurs/réparateurs; et les mêmes séquences : transgression, déplacement puis réparation, consolation, et retour à l'espace d'origine. Au cœur de chacune des histoires il y a une perte, un manque, dus en général à une transgression perpétrée par T'choupi, suivis à la fin de l'album d'une réparation ou d'une liquidation du manque. Et ce sont au fond toujours les mêmes valeurs qui sont mises en avant : la tension de l'enfant entre autonomie et dépendance. On se doute qu'un grand nombre d'album est construit sur le modèle narratif du conte et pourrait être analysé de la sorte. Retenons que le point déterminant dans ce modèle est l'aspect onomasiologique : il y a d'abord un récit que viennent ensuite mettre en scène les images. Elles peuvent être belles et bien présentes, le sens de 1'album n'est pas construit en priorité à partir de leurs propriétés. 


\section{2) Modèle de la boule de neige (dialectique sémasiologique)}

À l'inverse, les albums relevant du modèle de la boule de neige ne cessent de redécouvrir les vertus des images et de leur disposition particulière dans les albums. Comme dans l'exemple de La piscine, d'une page à l'autre les variations du plan de l'expression iconique impliquent des variations sur le plan du contenu, et plus précisément au niveau narratif. À chaque nouvelle page un élément supplémentaire apparaît, transformant l'album en une sorte de comptine jouant avec les charmes de la répétition. L'expression «boule de neige » traduit ce mécanisme qui déroule et répète à l'envie une cellule narrative toute simple. Et la boule, souvent, s'accroit de page en page jusqu'au dénouement final. Les statistiques restent à faire, mais il semblerait que ce soit le modèle le plus largement répandu, ce qui se comprend bien car à il tient compte de la spécificité visuelle de l'album tout en pouvant faire fonds sur une grande productivité. Autrement dit, nous allons rencontrer des progressions narratives récurrentes car très tentantes à partir de la disposition linéaire des albums. À partir du cadre général, plusieurs possibilités sont permises en fonction de ce qui varie et du type de variation. Trois sous modèles paraissent pouvoir incarner le modèle de la boule de neige : la déclinaison, l'intensification et la chaine.

La déclinaison représente le cas le plus simple où, d'une page à l'autre, un seul élément iconique varie, entraînant une variation narrative minime. On trouvera par exemple un même rôle tenu par différents acteurs, ou un acteur qui s'obstine à appliquer un programme narratif à de multiples objets. Le cas célèbre De la petite taupe qui voulait savoir qui lui avait fait sur la tête (Holzwarth, Erlbruch, 1993) décline une gamme à sa façon, sur le registre de l'enquête scatologique. La petite taupe cherche avec entêtement, de double page en double page, qui a osé lui « faire sur la tête » en première page. À chaque nouvelle image, l'interlocuteur change : pigeon, cheval, lièvre, chacun exhibant pour la taupe ce qu'il fait comme excrément, preuve de son innocence. Les variations aboutissent enfin à l'identification du coupable et à la terrible vengeance de la taupe. Citons pour exemple, dans le même registre Et pourquoi, de Michel Van Zeveren (2007) où un imperturbable petit chaperon rouge vient à bout d'un grand loup gris à force de répéter un «Et pourquoi » qui conduit le loup au suicide. Pour rester en compagnie des loups, citons aussi « C'est moi le plus fort » (Ramos, 2001), où la créature demande confirmation à chaque animal rencontré de son indéniable supériorité.

L'intensification exploite le même principe, mais en lui adjoignant une gradualité : l'une des dimensions événementielles est soumise à une intensité croissante ou décroissante. C'est par exemple une classe d'acteur remplissant un rôle identique qui peut être articulée ainsi, comme dans le cas de La piscine, où l'on va de la plus petite des créatures à la plus grosse. Le ressort narratif est à peu près identique dans Nuit noire (Dorothée de Monfreid) : Fantin, un petit garçon est perdu dans les bois la nuit, il a peur. Il entend du bruit, se cache et voit un loup qui s'approche et se réchauffe devant un feu de bois ; le loup est bientôt chassé par un tigre, qui sera chassé à son tour par un énorme crocodile. Heureusement, dans le creux de l'arbre vit un lapin, qui trouve un subterfuge pour quitter la forêt : se déguiser en une créature encore plus grande et plus effrayante que celles rencontrées auparavant.

Autre possibilité, la chaine : ce cas de figure se rapproche de la déclinaison en lui conférant un tour plus dynamique, et parfois cyclique. La cellule narrative se maintient, mais les acteurs changent de rôle d'une page à l'autre : par exemple A demande à $\mathrm{B}$, qui demande à $\mathrm{C}$, etc. Plouf de Philippe Corentin (1991) illustre 
bien cet effet de réaction en chaîne : un loup affamé croit voir la lune dans un puits, tombe dans le puits et découvre que ce n'était que le reflet de la lune. Page suivante, viennent trois cochons, auquel le loup fait miroiter le beau fromage. « Hop, les cochons descendent dans le seau » et le loup remonte dans le même temps, sans pouvoir attraper les cochons. Les cochons abuseront à leur tour de trois petits lapins, auxquels on a fait miroiter l'existence de carottes au fond du puits. Enfin le loup, retombe dans le travers initial, attiré non plus par une fausse lune mais trois vrais petits lapin : il se penche, tombe, les lapins remontent et le loup se retrouve au fond du puits.

On peut clore l'examen de ce modèle de la boule de neige par deux remarques : le plus souvent les trois variantes sont susceptibles d'interagir dans les albums, l'une prenant le relais de l'autre ou offrant la possibilité d'un dénouement surprenant, ou prévisible. Par exemple, dans C'est moi le plus fort, après déclinaison de la formule devant chaque animal, l'album se clôt par un saut relevant de l'intensification : le loup croise une sorte de faible petit crapaud tout vert, qui ose répondre que c'est sa maman, la plus forte. Et d'ailleurs elle est là, juste derrière lui, qui s'avère être un bébé dragon. L'album finit sur cette phrase emblématique de notre modèle : «Moi ? Moi..., je suis le petit gentil loup ».

Dernière remarque, ce modèle apparemment simple peut être l'occasion de formes narratives très inventives. Le modèle générique ne limite pas l'œuvre, il la nourrit. Dans Cependant ... Le livre le plus court du monde (2001), Paul Cox déploie une structure étonnante. Ce beau livre d'art et néanmoins album efficace, se plie merveilleusement aux règles du genre que nous avons établies. L'ouvrage n'a ni début ni fin, autrement dit, pas de couverture, mais une disposition linéaire incontournable, cyclique, qui donne sens à l'album. En haut à gauche de chaque illustration figure un énigmatique « cependant», qui surplombe une illustration représentant une action, sans que l'on puisse établir clairement de lien entre le texte et l'image ni de lien, en dehors de la présence du « cependant», entre deux images. Une lecture plus attentive permet de découvrir que la lumière croit ou décroit régulièrement d'une illustration à l'autre, accomplissant un parcours allant de la lumière la plus forte à la nuit la plus noire. L'album invite le lecteur à accomplir le tour de la terre en arrêtant le temps et à découvrir ce que font les habitants de ces différents lieux au même moment. Autrement dit, page après page varient : l'acteur qui fait l'action, l'action elle-même, l'espace et la lumière. Demeurent la représentation d'une action (aller sur la lune, se coucher, manger, etc.), et la localisation temporelle, identifiée par le « Cependant » répété à chaque nouvelle page. Cette suspension du temps et ce parcours dans l'espace au fil de l'œuvre montrent de manière assez exemplaire ce que peut être l'essence de l'album ou, dans un vocabulaire plus technique, sa généricité.

\section{3) Modèle de la surprise (dialogique)}

Le modèle de la surprise, d'essence énonciative, avec ses variantes, est également très productif dans les publications actuelles, notamment parmi les œuvres les plus élaborées. Dans ce cas l'ensemble du texte est construit sur une manipulation du point de vue, capable de transformer le sens de tout ce qui a été raconté antérieurement. Là encore on doit distinguer trois voies destinées à susciter la surprise du lecteur d'album, qui jouent chacune sur un décalage particulier. Le premier cas relève de ce que l'on peut appeler une "surprise énonciative » lorsque c'est l'univers de référence de l'observateur qui domine, le décalage ne prenant sens que de ce point de vue de l'observateur. Le second cas se traduit par une rup- 
ture énonciative. L'univers de référence des acteurs et du narrateur de l'énoncé impose alors son point de vue à l'observateur. Enfin l'effet peut venir de «tensions énoncives ", lorsque le décalage de point de vue oppose des acteurs de l'énoncé lui-même, sans recours à l'observateur extérieur. Claude Ponti semble être un pratiquant convaincu et convaincant des deux premiers types ; il nous fournira donc la matière pour les illustrer et expliquer leur fonctionnement par l'exemple.

La surprise énonciative peut s'observer par exemple dans « Au fond du jardin ». Ce texte appartient à un album intitulé Dans la voiture. Au fond du jardin. Sur la branche (Ponti, 1996) mettant en scène trois courts scénarios où l'image joue un rôle décisif. La première double page nous dévoile le fond du jardin. Et ce matin, nous dit le texte en bas de page, " au fond du jardin, une souris cueille une fraise sauvage pour son amie » et « deux coccinelles se réveillent». Au centre de l'image trône un melon, à gauche, au niveau du sol apparaissent les oreilles de la souris derrière deux fraises, alors que les deux coccinelles sont cachées dans les feuilles à droite. Deux papillons sont posés sur une branche. Dans l'image qui suit apparaît un oiseau qui semble vouloir se poser sur le melon, alors que souris, coccinelles et papillons poursuivent leur activité. Le moment de la surprise arrive avec l'image suivante : un court instant, enregistré par l'image, le melon prend vie, la fraise lancée par la souris de droite à une souris sur la droite lui fait un nez rouge, les coccinelles marquent les yeux, les papillons deux oreilles convenables et l'oiseau, qui a vaguement une tête de canari, dessine la chevelure. Dans l'image suivante le temps a suivi son cours, le melon est redevenu un melon inanimé. Ce moment d'unité n'a de sens, au premier abord, que pour l'observateur extérieur, seul capable de saisir le moment de vie du melon. Il s'agit bien d'une surprise énonciative, où le point de vue de l'observateur donne sens à l'ensemble, les acteurs de l'énoncé étant a priori indifférents à ce court moment de grâce.

La rupture énonciative agit différemment car, contrairement au premier sous modèle, le narrateur se joue le plus longtemps possible de l'observateur, jusqu'à la surprise et la révélation de la supercherie. Le point de vue factuel, où les choses peuvent être décrites comme vraies, appartient alors aux acteurs de l'énoncé et au narrateur. Tromboline et Foulbazar sont, dans ce registre, deux poussins toujours très surprenants. Par exemple dans Le chat et le chien (Ponti, 2007), on voit Tromboline s'amuser avec un chien, puis Foulbazar faire de même avec un chat, puis le chat et le chien qui jouent de conserve, sans jamais voir les quatre acteurs ensemble. À ce stade, l'observateur peut avoir quelques doutes légitimes sur ce qu' on est en train de lui montrer, mais il reste tributaire de ce que vont dévoiler les acteurs. En dernière page le lecteur découvre avec stupeur les deux poussins en train de remiser leur déguisement, ce qui oblige à reconsidérer l'ensemble de ce qui vient d'être lu : il n'y avait pas quatre acteurs, mais deux acteurs, d'où l'impossibilité de voir Tromboline et Foulbazar jouer avec le chat et le chien. Ce type de manipulation est particulièrement habile; il invite l'enfant observateur à adopter une position critique puisqu'il est amené à réfléchir au statut de ce qui vient de lui être lu et montré.

La tension énoncive est encore une façon de mettre en scène un décalage. Loulou, de Solotareff (2001) en est un exemple typique. Un loup orphelin et un lapin solitaire deviennent amis. Le lapin apprendra au loup à pécher pour se nourrir, tandis que le loup enseignera au lapin l'art de courir aussi vite qu'un loup. La rupture advient lorsqu'ils jouent à faire peur, « PEUR-DU-LOUP » étant beaucoup plus efficace que «PEUR-DU-LAPIN»! Une tension prend corps entre le monde possible du loup et le monde possible du lapin. L'univers réunissant le loup et le lapin devient 
contrefactuel, à la fois du point de vue de chacun des acteurs loup et lapin et du point de vue de l'observateur. La surprise est interne : elle appartient aux acteurs de l'énoncé. Le lapin est surpris du comportement du loup, de l'efficacité de la «PEUR-DU-LOUP », et de la nature qui reprend ses droits, alors qu'il y a plutôt, du point de vue de l'observateur une attente. Le lecteur sait que même dans un album lapin et loup font rarement bon ménage.

\section{Arbre générique des albums d'enfance}

L'ensemble de ces déterminations successives dessine l'arbre générique des albums d'enfance, qui représente la hiérarchie entre composantes textuels imposées par le genre de l'album d'enfance.

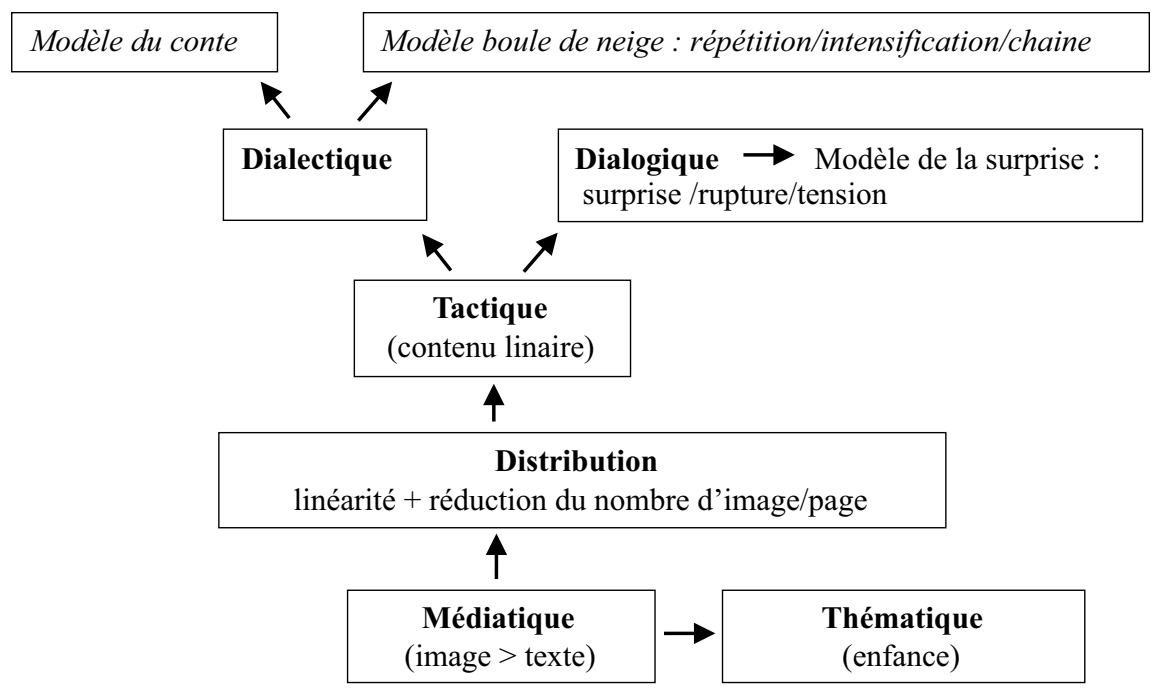

Hiérarchie des composantes textuelles dans l'arbre générique:

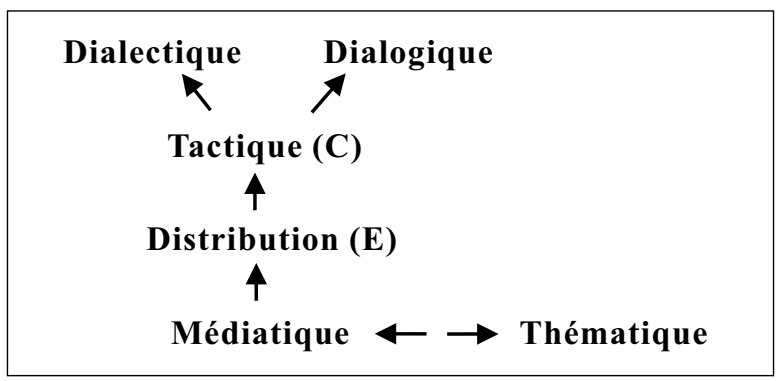

\section{De l'arbre au gradient : les voies de l'œuvre}

En guise d'ouverture finale, plus que de conclusion, on peut tenter d'envisager l'autre versant de la généricité, précédemment décrite à partir des albums, et les implications théoriques d'une mise en perspective de la singularité à partir de la généricité. 
La sémantique textuelle revendique une hétérarchie des composantes textuelles, qui s'oppose notamment à la générativité au cœur de la sémiotique greimassienne. Il n'y a pas pour le point de vue sémantique, une catégorie élémentaire à partir de laquelle serait engendrée le texte par enrichissements successifs. Les composantes apparaissent au contraire comme des interactions spécifiques, propre à chaque occurrence textuelle. La prise en compte du genre, défini comme hiérarchie des composantes, nuance le propos, et tout se passe comme si la généricité tenait le rôle de la générativité, en substituant à la pure justification logique un déterminisme à la fois social et sémantique. Mais une différence fondamentale demeure entre le modèle génératif et le modèle générique qui permet à la généricité d'éviter l'un des gros écueils de la générativité. Dans la générativité, la conversion d'un niveau dans l'autre devrait permettre d'aboutir logiquement et de manière entièrement autonome au résultat final, qu'il s'agisse d'un texte ou d'un discours. Or, dans le modèle sémiotique, l'engendrement échoue à produire le discours final de manière autonome car manque une théorie de la conversion, expliquant le passage d'un niveau à l'autre. Ce constat est d'ailleurs bien assumé en sémiotique greimassienne, où l'on reconnaît volontiers que la conversion demeure en partie mystérieuse ${ }^{(12)}$.

Rien de tel dans le modèle générique que nous venons de déployer car, d'une part, il ne s'agit que d'un ensemble de prescriptions et de normes et que d'autre part, ces prescription génériques ne pèsent pas toutes d'un même poids. Il y a, de droit, dans ce modèle, place pour un sujet, pour un auteur qui trouve le chemin de l'œuvre à partir des contraintes génériques.. Du socle générique aux combinaisons envisageables à partir du socle jusqu'à la singularité des productions textuelles, s'ouvre un gradient, qui va du plus générique au moins générique, de la coercition la plus forte à la liberté la plus grande. D'un degré à l'autre, le poids de la contrainte générique se fait moindre alors que parallèlement les possibilités de singularisation offertes à l'auteur — proportionnelles à l'étendue des choix possibles - s'accroissent. Cette perspective conduit à reformuler notre arbre générique des albums comme un gradient de généricité, où les différents degrés se déploient entre les pôles de la généricité et de la singularité. La présente étude ne portait que sur la dimension la plus générique, en montrant néanmoins déjà comment la palette des choix possibles se déployait à partir du socle génériques. En continuant à parcourir le gradient en direction du pôle de la singularité on doit ménager la possibilité de degrés, telle que la combinaison entre modèles notamment, aboutissant au style puis à l'œuvre la plus singulière.

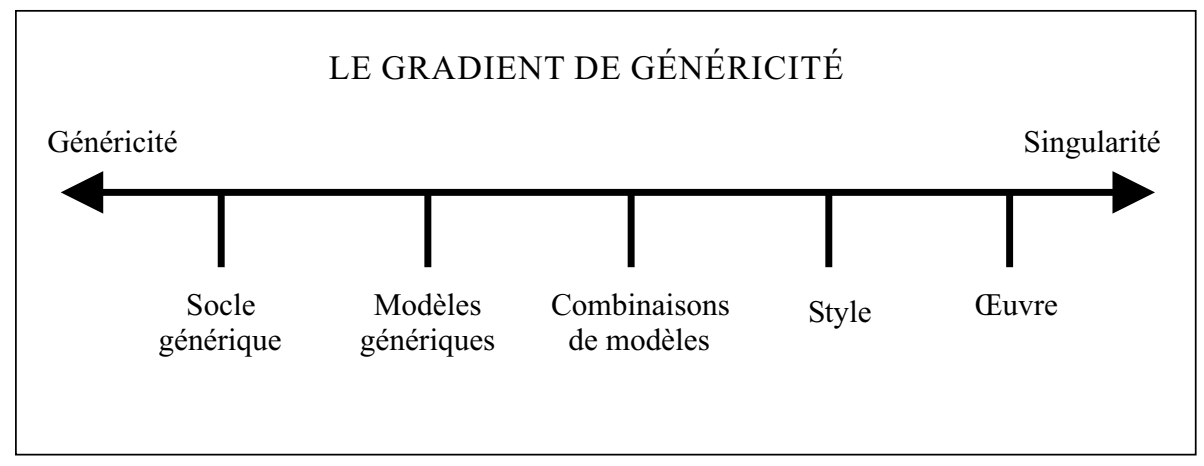

(12) Par exemple Fontanille 2001. 
Du genre aux œuvres le poids de la généricité décroit jusqu'à paraître s'effacer en partie devant l'invention du style et des œuvres. Mais la généricité ne disparaît jamais totalement; elle reste, en synchronie, 1'horizon sur lequel l'œuvre se détache. François Rastier dans Art et sciences du texte définit le style comme une interaction idiolectale entre composante textuelle, alors que le genre consacre une interaction de nature sociolectale. On peut ajouter, pour conclure et ouvrir sur le pôle inverse de la généricité, que l'interaction idiolectale consiste nécessairement pour partie en une appropriation de l'interaction sociolectale sélectionnée préalablement par l'appartenance à un genre particulier. Reste alors à montrer par une analyse stylistique, en quoi consiste le jeu qui s'offre à l'auteur, ses latitudes et ses contraintes dans l'interaction idiolectale des composantes textuelles. C'est là sans doute, le fin mot d'une réflexion sémiotique sur le genre qui entend s'inscrire dans une sémiotique des cultures, car : « de même que les normes sociales constituent le fond qui permet de comprendre les actions individuelles, l'étude du genre revêt son plus grand intérêt quand elle permet de percevoir la singularité des textes $\gg{ }^{(13)}$.

(13) F. Rastier, «Éléments de théorie des genres », op. cit. 


\section{Bibliographie}

ADAM, J.-M, HEIDMANN, U. (2004) : «Des genres à la généricité : L'exemple des contes (Perrault et les Grimm) », Langage.

Chelebourg, Ch., Marcoin, F. (2007) : La littérature de jeunesse, Paris, Armand Colin, coll. 128.

CoUÉGNAS, N. (2010) : «L'art de la surprise et de la répétition dans la littérature d'enfance et de jeunesse », dans Répétition et habitude dans les pratiques quotidiennes, dirigé par J. Alonso et E. Bertin, revue Protée, Université du Québec à Chicoutimi, volume 38, $\mathrm{n}^{\circ} 2$.

FontAnille, J. (2001) : «La sémiotique est-elle générative ? », M. Arrivé \& S. Badir, dir., Spécificité et histoire des discours sémiotiques, Linx 44.

RAstier, F. (1989) : Sens et textualité, Paris, Hachette.

- (1994) : Sémantique pour l'analyse. De la linguistique à l'informatique, Paris : Masson.

- (2001): Arts et sciences des textes, Paris, PUF.

— (2001) : «Éléments de théorie des genres », Texto ! juin 2001 [en ligne]. Disponible sur : http://www.revue-texto.net/Inedits/Rastier/Rastier_Elements.html. (Consultée le 25-05-2012).

VAN DeR Linden, S. (2006) : Lire l'album, Le Puy-en-Velay, L'atelier du poisson soluble.

\section{Albums cités}

Corentin, P., Plouf, Paris, L'école des loisirs, 1991.

Courtin, T., T'choupi se perd au super marché, Paris, Nathan, 1999.

CoX, P., Cependant... Le livre le plus court du monde, Paris, Seuil, 2002.

Edy-Legrand, E., 1919, Macao et Cosmage, Paris, Éd. Circonflexe, 2000.

Got, Max le crocodile, Paris, Éd. du Seuil, 1995.

HOLZWARTH, W, ERLBRUCH, W, 1989, De la petite taupe qui voulait savoir qui lui avait fait sur la tête, Paris, Milan, 1993.

MonfREID (DE), D., Nuit noire, Paris, L'école des loisirs, 2008.

PONTI, C., Tromboline et Foulbazar. Le chat et le chien, Paris, L'école des loisirs, 2007.

PONTI, C., Dans la voiture. Au fond du jardin. Sur la branche, L'école des loisirs, 2000.

Poussier, A., La Piscine, Paris, Éd. L'école des loisirs, 2008.

RAmos, M., C'est moi le plus fort, Paris, Pastel, 2001

SolotarefF, G., Loulou, L'école des loisirs, 2001.

VAn ZeVeren, Et pourquoi, Paris, Pastel, L'école des loisirs, 2007. 